\title{
La minière Néolithique ancien / moyen I des Longrais à Soumont-Saint-Quentin (Calvados)
}

The Ancient/Middle Neolithic Les Longrais flint mine at Soumont-Saint-Quentin (Calvados)

Jean Desloges, Emmanuel Ghesquière et Cyril Marcigny

Traducteur : François Charraud

\section{(2) OpenEdition}

\section{Journals}

Édition électronique

URL : https://journals.openedition.org/rao/1306

DOI : 10.4000/rao.1306

ISSN : 1775-3732

\section{Éditeur}

Presses universitaires de Rennes

\section{Édition imprimée}

Date de publication : 31 décembre 2010

Pagination : 21-38

ISBN : 978-2-7535-1383-9

ISSN : 0767-709X

\section{Référence électronique}

Jean Desloges, Emmanuel Ghesquière et Cyril Marcigny, « La minière Néolithique ancien / moyen I des Longrais à Soumont-Saint-Quentin (Calvados)», Revue archéologique de l'Ouest [En ligne], 27 | 2010, mis en ligne le 25 février 2012, consulté le 18 août 2022. URL : http://journals.openedition.org/ rao/1306; DOI : https://doi.org/10.4000/rao.1306

Ce document a été généré automatiquement le 18 août 2022.

Tous droits réservés 


\section{La minière Néolithique ancien / moyen I des Longrais à Soumont-Saint-Quentin (Calvados)}

The Ancient/Middle Neolithic Les Longrais flint mine at Soumont-Saint-Quentin (Calvados)

Jean Desloges, Emmanuel Ghesquière et Cyril Marcigny

Traduction : François Charraud

1 L'implantation d'un lotissement à Soumont-Saint-Quentin, au lieu-dit «Les Menhirs » ou «les Longrais", a nécessité la réalisation d'un diagnostic archéologique (sur $8500 \mathrm{~m}^{2}$ ) en février 2008 (fig. 1), à proximité immédiate de la fouille conduite par B. Edeine dans les années 1960 sur des minières de silex et des sépultures du Hallstatt (Edeine, 1961, 1963, 1965 a et b, 1972).

1. LES MINIÈRES DE SILEX DE LA DELLE DES LONGRAIS : RETOUR SUR UN SITE EMBLÉMATIQUE

3 Avant de préciser les résultats de ce diagnostic, il n'est pas superflu de faire un point sur son contexte géologique, archéologique et historique. Il est en effet impossible d'évoquer le site des Longrais sans le rattacher à son environnement immédiat ; celui-ci est sans équivalent et mérite le qualificatif de « haut lieu » au sens ou l'entendait Pierre Nora (1997). 

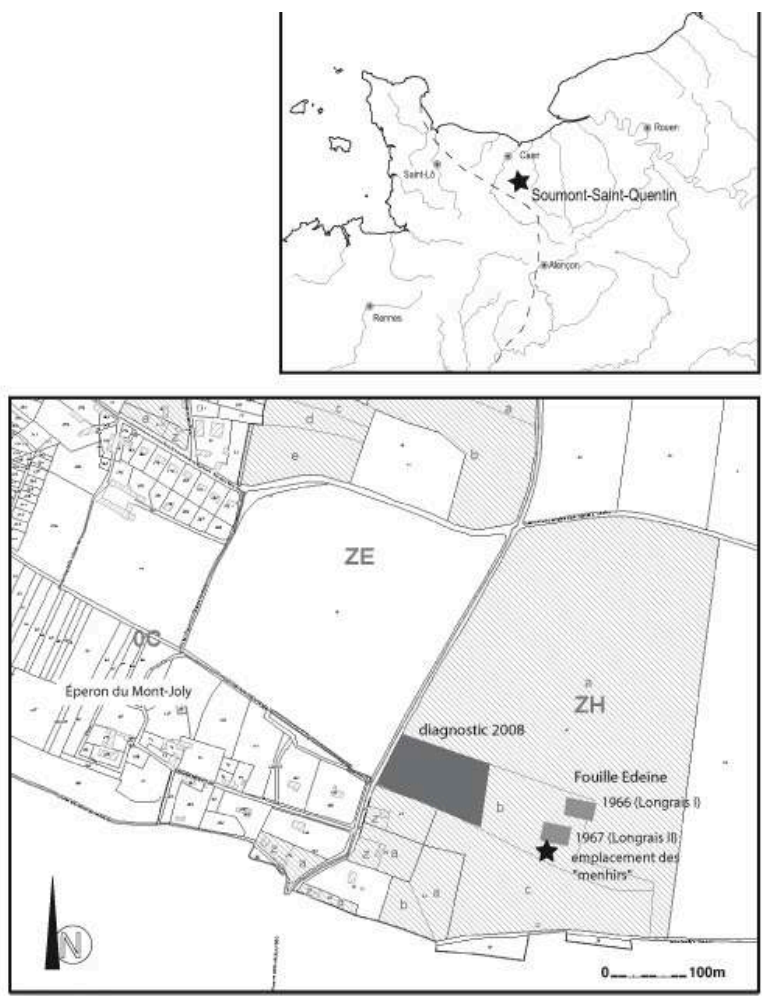

Figure 1 : Localisation du site des Longrais. Implantation de la zone diagnostiquée en 2008 par rapport aux fouilles de B. Edeine (en hachuré les parcelles constructibles). L'opération de 1966 est recalée d'après les archives de $B$. Edeine, celle de 1967 d'après une anomalie phytologique observable en photo aérienne et l'emplacement des menhirs d'après la description du site publiée dans les années 60 (DAO, C. Marcigny, Inrap).

Figure 1: Location of Les Longrais and plotting of the area studied in 2008 against previous interventions by B. Edeine (areas planned to be built are hatched). 1966 excavation are located from Edeine's Archives, 1967 oness from a crop-mark visible on air photography and menhirs' site from a description published in the 60 's.

\section{Un voisin éminent, l'éperon du Mont Joly}

L'éperon du Mont Joly est une de ces fantaisies de la nature qui ont de tout temps captivé les hommes. En termes géologiques, c'est un chaos de roches anciennes qui émerge brusquement au beau milieu de la plaine jurassique (fig. 2). Un modeste cours d'eau, le Laizon, s'est frayé un chemin aux dépens des grès, formant une cluse profonde de trente mètres : la Brèche au Diable. Sur sa rive est, le Laizon a découpé un véritable promontoire bordé d'abrupts sur trois côtés. Le sommet est constitué par une plateforme rabotée d'une superficie d'environ quatre hectares. L'archéologue y reconnaît un retranchement d'éperon et n'a aucune peine à identifier une barre talutée de $150 \mathrm{~m}$ de long à l'opposé de la cluse, c'est-à-dire là où le bombement de grès disparaît sous les calcaires (fig. 3).

5 C'est ici que les épigones d'Arcisse de Caumont, Frédéric Galeron et les premiers préhistoriens normands comme MM. Costard et Foucher ou Raoul Doranlo, écrivirent quelques pages fameuses de l'archéologie bas-Normande. Leurs trouvailles firent les beaux jours du musée de Falaise, malheureusement détruit en 1944. Pour l'anecdote, le potentiel archéologique des lieux fut révélé fortuitement en 1798 lors de la construction au bord de l'abrupt du tombeau de Marie Joly, célèbre pensionnaire de la 
Comédie française et l'une des égéries du Romantisme. La Roche-Saint-Quentin devint alors pour tous le Mont Joly (Doranlo, 1926).

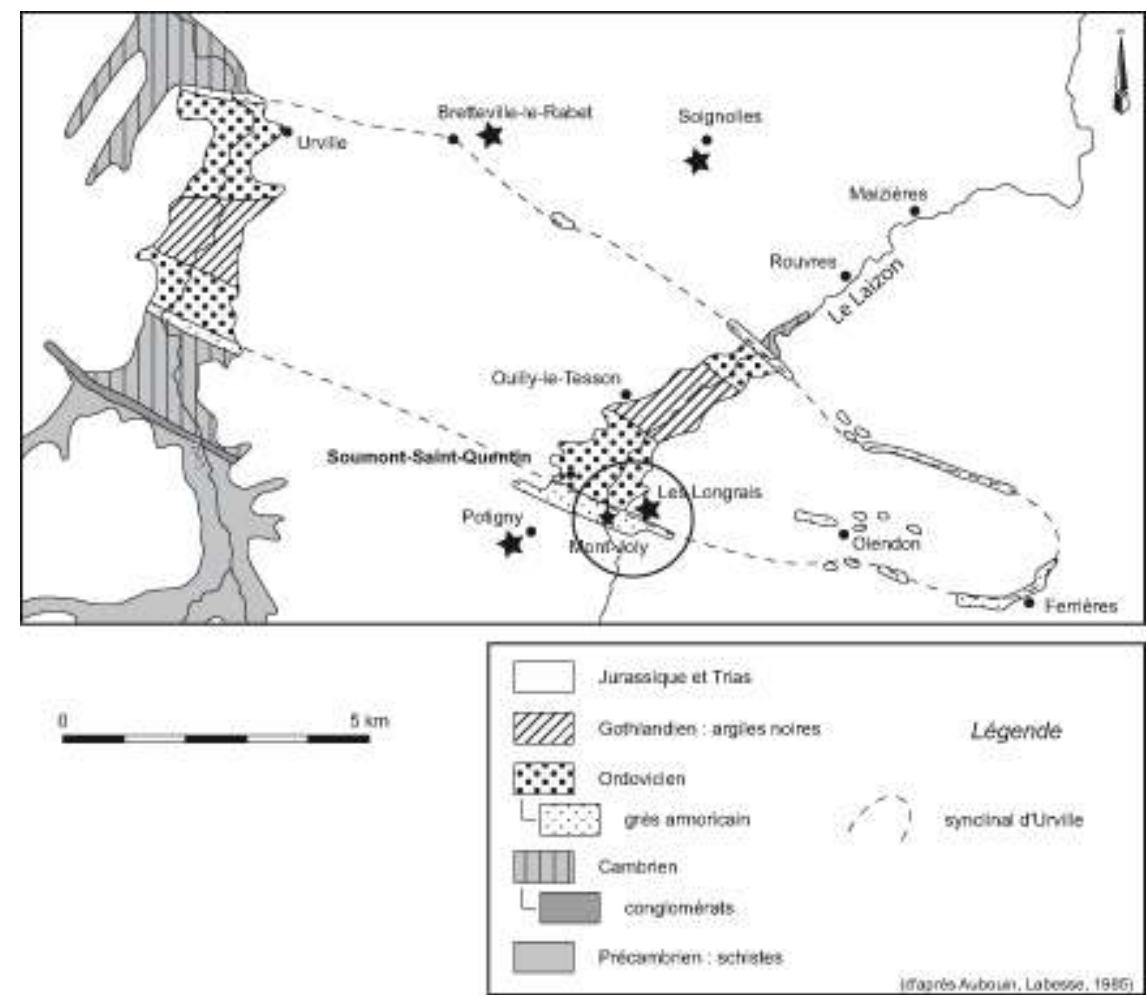

Figure 2 : Carte géologique simplifiée de la région de Soumont-Saint-Quentin, le site (étoile) est implanté sur le substrat calcaire en bordure des affleurements de grès (DAO, B. Van den Bossche, Paris I).

Figure 2: Simplified regional geological map. The site (star) is on jurassic limestone, but very close to Ordovician sandstone outcrops. 


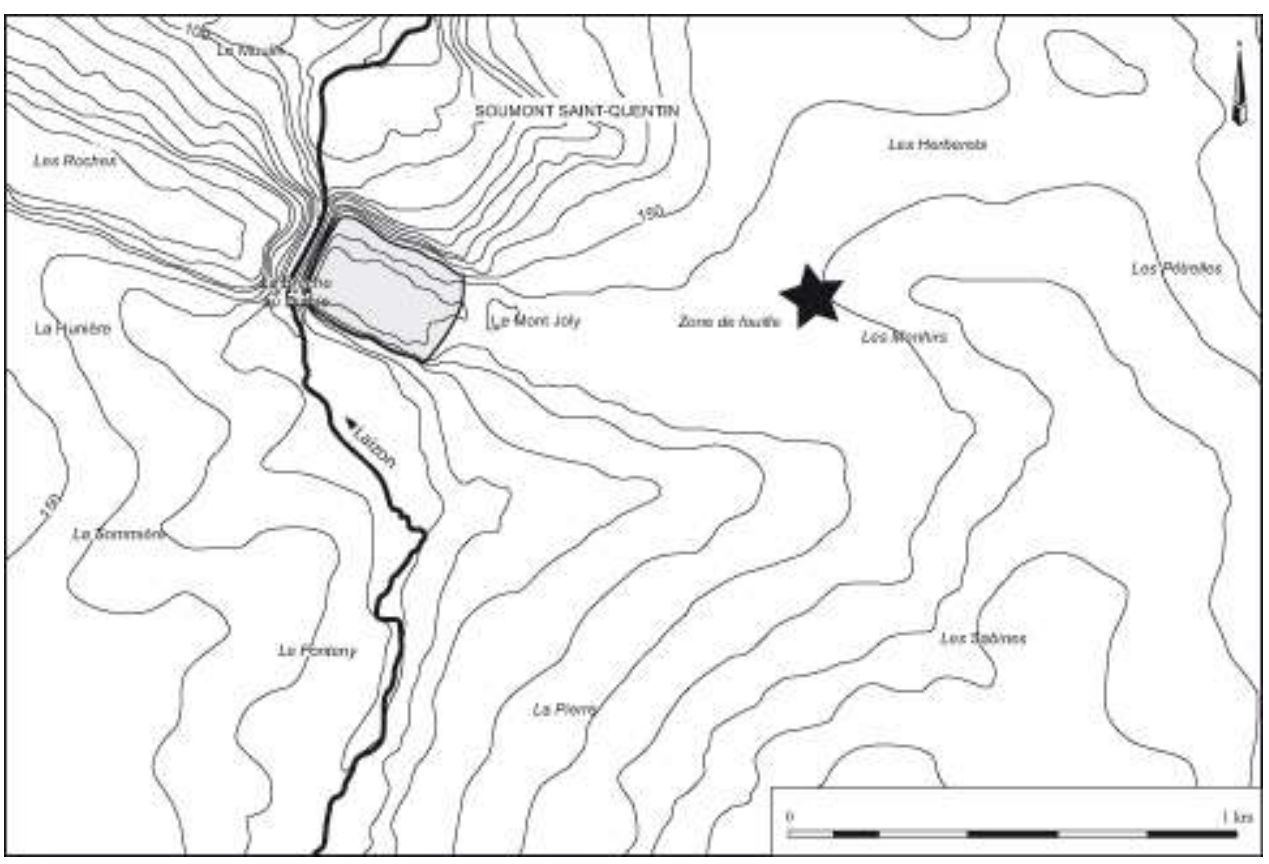

Figure 3 : Localisation géographique et contexte topographique du Mont-Joly. La trame grisée indique l'emprise approximative de l'habitat ceinturé par le rempart, le champ des « Menhirs » est dans le même axe vers l'est (DAO, B. Van den Bossche, Paris I)

Figure 3: Geographical location and topographic environment of Le Mont Joly. Tinted area marks the approximate extent of rampart-surrounded occupation; the "Menhirs' field" is on the same axis, eastwards.

C'est à partir des années 1910 que des blocs polissoirs sont identifiés au pied de l'éperon, confirmant une fréquentation néolithique que l'industrie en silex montrait déjà en abondance. Une nouvelle étape de la recherche archéologique s'ouvre à partir de 1955 : la richesse des vestiges mis à découvert par des travaux de génie civil suscite quelques velléités et Bernard Edeine, formé à l'ethnologie, se propose de mettre en œuvre les méthodes archéologiques initiées par André Leroi-Gourhan avec la notion de «Palethnologie ». Avec l'aide d'amateurs de haut niveau parmi lesquels Robert Caillaud et Edouard Lagnel, il entreprend une campagne de sondages en différents points. L'intérêt, qui se portait au gré des découvertes sur l'occupation protohistorique et le cimetière mérovingien, se déplace brusquement vers le Néolithique. La publication de la thèse de Gérard Bailloud dans les années soixante en est la cause évidente (Bailloud, 1964, p. 399-429) : les fouilles du Mont Joly arrivent à point nommé pour conforter sa périodisation, mais les arguments scientifiques sont bien maigres et aucune publication sérieuse ne vient les étayer. Finalement les autorisations de fouilles ne seront pas renouvelées.

7 Aujourd'hui, il reste peu de données concrètes sur les fouilles alors pratiquées au Mont Joly. La documentation scientifique subsistante est disparate et peu utilisable. Pour résumer, le site ressemble à un condensé de l'archéologie métropolitaine, depuis les industries du Paléolithique inférieur jusqu'aux sépultures du haut Moyen Âge. La période néolithique, qui nous intéresse ici, est représentée par des éléments domestiques où on peut reconnaître les styles céramiques propres au Néolithique moyen, Cerny et Chasséen. Le retranchement est en revanche daté de l'âge du Bronze mais tout porte à penser que cet ouvrage reprend une levée plus ancienne. 


\section{Les Longrais et les minières de silex}

8 Le site des Longrais se trouve en quelque sorte accolé à l'éperon du Mont Joly ; plus précisément, il occupe le plateau calcaire qui enserre l'extrémité orientale du renflement de grès. Cette terre de labour attira très tôt l'attention des collectionneurs qui pouvaient y recueillir en surface une profusion de silex. La découverte survient en plein mouvement "Campignien " (Salmon et al., 1898), et en a tous les caractères. Le site est vite rattaché à un ensemble plus vaste, celui d'Olendon (Eudes-Deslongchamps, 1876). L'arrière-plaine du Mont Joly est alors couverte de ces silex à tel point que la Société préhistorique française crée «l'olendonien» dans la chronologie du "Néolithique inférieur ». La reconnaissance du phénomène, pour éphémère qu'elle ait été, eut le mérite de révéler pour la première fois l'un des caractères originaux du Néolithique bas-normand. Si l'on en croit la chronique, le site des Longrais était réputé pour livrer des quantités d'éclats, d'où le qualificatif d'atelier, mais son originalité venait du fait que les tranchets étaient les seuls outils que l'on y trouvait. À $3 \mathrm{~km}$ de là, Olendon, au contraire, n'offrait que des pics et des « haches grossières ».

En 1955, un événement va permettre indirectement de confirmer la nature du site : le propriétaire des Longrais vient de recueillir un curieux objet en bronze. Bernard Edeine décide d'abandonner momentanément le Mont Joly et lance une opération de fouille aux Longrais (fig. 1) et trois riches sépultures du Hallstatt sont découvertes (Edeine, 1961). Ce n'est que dix années plus tard que l'équipe de Bernard Edeine investit à nouveau les Longrais. Au lieu des sépultures attendues, c'est un étrange semis de fosses et des amas de silex qui se présentent sur le substrat décapé au bulldozer. D'abord qualifiées de foyers, puis d'ateliers, ces structures se révèlent finalement être des puits d'extraction de silex dont la profondeur ne dépasse pas 1,50 m (fig. 4, 5, 6). L'analogie avec les structures d'extraction fouillées jadis par G. Fouju dans l'Oise ne laissait pas de doute sur la nature du site. Bernard Edeine fit relever les profils des comblements de plusieurs cavités, mais les fouilles ne furent pas poussées plus avant. Quant aux " amas" de silex, plusieurs ont fait l'objet d'un relevé graphique mais les pièces non sélectionnées furent jetées dans une décharge sauvage. Les fouilleurs de l'époque se souviennent que la quantité de silex taillé était relativement faible et ne concernait visiblement que la fabrication de tranchets. Cependant, quelques nucléus à lames ont été également recueillis dans les mêmes conditions. Bernard Edeine consacre d'ailleurs plusieurs notes au matériel des Longrais qu'il qualifie de "danubo-campignien" (Edeine, 1963, 1970 et 1972). Par la suite, il cherchera à faire l'impasse sur la présence de puits d'extraction tant le rapprochement avec le Néolithique ancien lui semble saugrenue. Des datations radiocarbone sont toutefois là pour confirmer l'ancienneté du site (en particulier la date Gif 2315 : 6490+/-160 BP - foyer F3 niv. 50 - obtenue sur un charbon de bois découvert avec la céramique $\mathrm{n}^{\circ} 1$ de la fig. 7). Et surtout, les fouilles permettent de recueillir quelques tessons décorés dans le "style danubien ", décors attribués depuis au Blicquy - Villeneuve-Saint-Germain et au Cerny (fig. 7). 


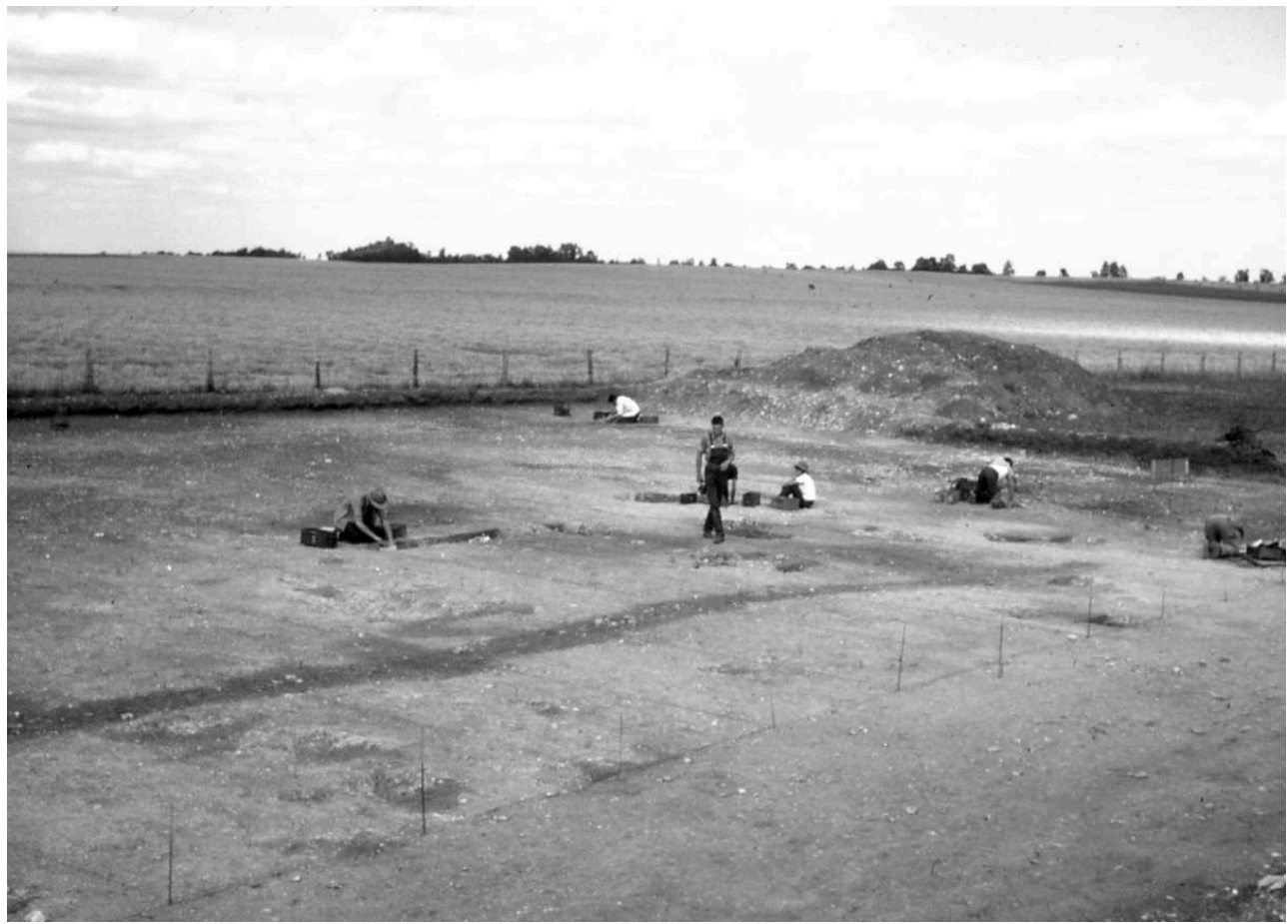

Figure 4 : Vue générale de la fouille de 1966 ; on aperçoit au sol l'ouverture des puits de mine, alors en cours de fouille (photo J.-P. Watté).

Figure 4: General view of 1966 excavation ; mouth of mine pits, then in course of excavation, are visible on scraped surface.

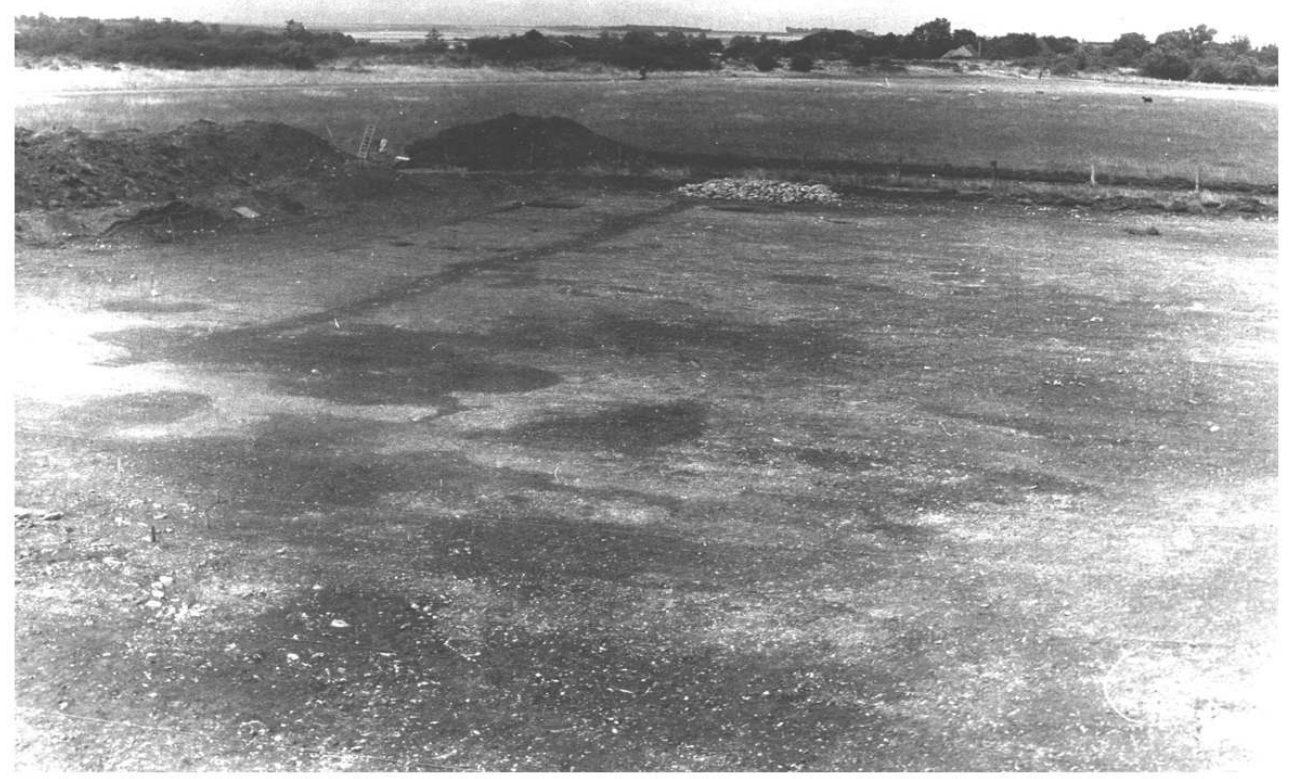

Figure 5 : Le secteur des Longrais en 1966 après décapage à la pelle mécanique ; les puits sont bien visibles à gauche du cliché (archives B. Edeine déposées au SRA de Basse-Normandie).

Figure 5: Les Longrais area after mechanical scraping in 1966; pits are clearly visible on the right. 


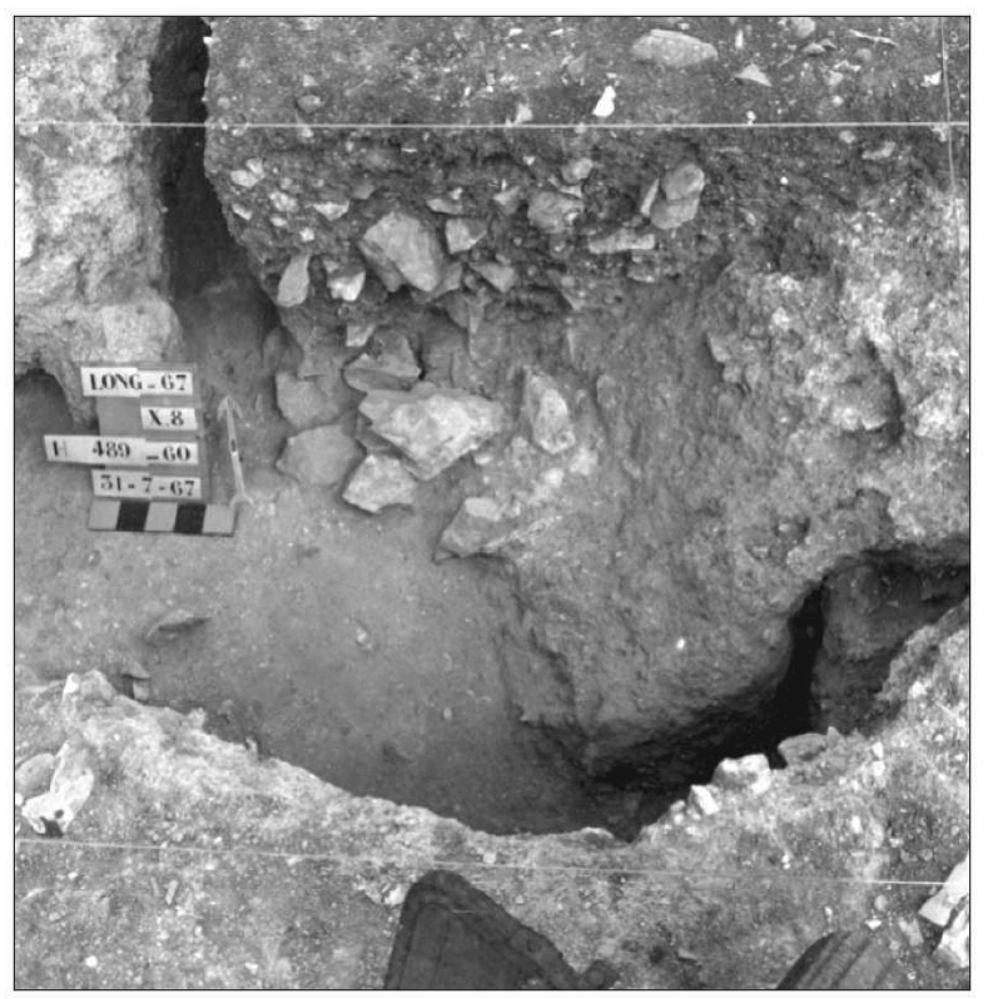

Figure 6 : En 1967, un des puits en cours de fouille (archives B. Edeine déposées au SRA de Basse-Normandie).

Figure 6: In 1967, one of the pits during excavation under B. Edeine's direction.

11 Dans le courant des années 60, d'autres puits à silex sont identifiés fortuitement dans cette même zone, à Soignolles et Potigny, mais ce n'est qu'en 1980 qu'un programme de fouilles spécialement consacré aux minières de silex est mis sur pied. On choisit un site «neuf », celui de Bretteville-le-Rabet, identifié par J. Desloges en 1982 à une dizaine de kilomètre au nord des Longrais. Cette opération pionnière s'échelonne jusqu'en 1987 (Desloges, 1990, 1999). En renouvelant les problématiques, elle ouvre en quelque sorte la voie aux grands chantiers de l'archéologie préventive des années 90 (Jablines, Serbonnes, Villemaur etc.).

12 À son tour, la Basse-Normandie a pu tout récemment bénéficier des moyens de l'archéologie préventive en ce domaine. La création de l'autoroute Falaise-Sées a été l'occasion en 2007 de reprendre la problématique minière régionale, l'emprise routière devant traverser un site inédit considéré comme un lieu d'extraction et de taille de haches. Cette fouille, à Ri-et-Ronai, près d'Argentan, a permis d'étudier une superficie de deux hectares offrant pour la première fois l'étude intégrale de plus de 600 puits d'extraction (Marcigny et al., 2007). 

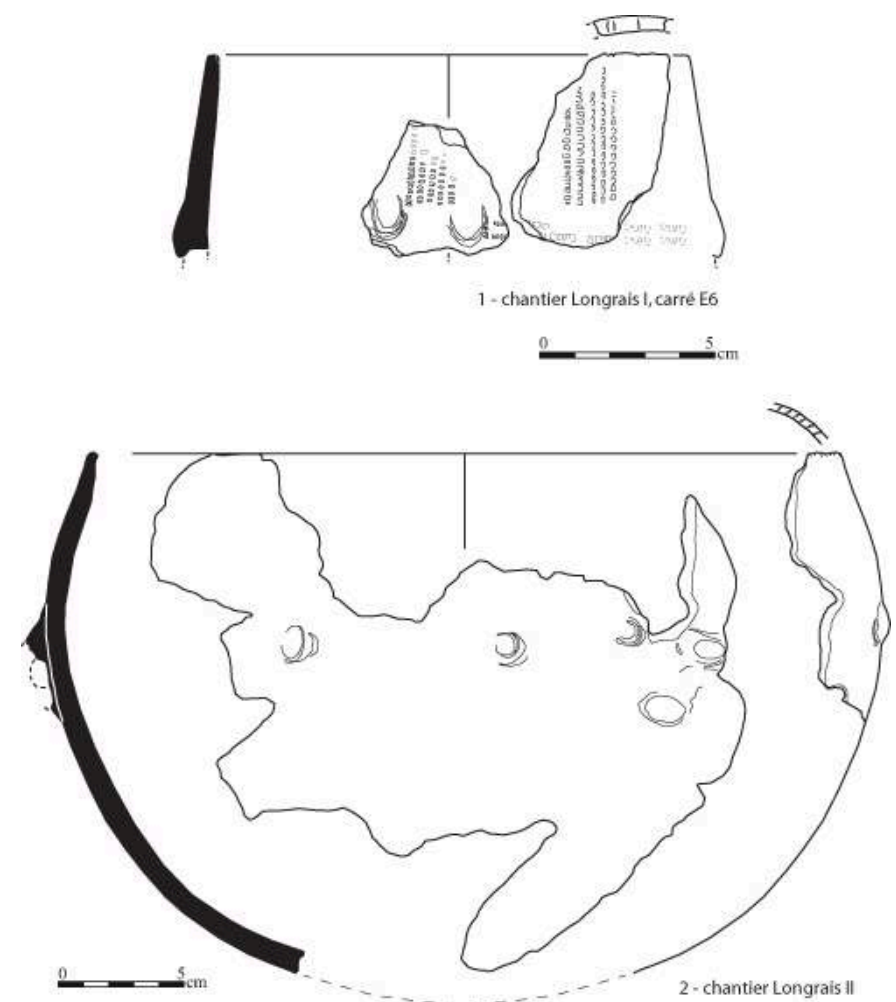

Figure 7 : Mobilier céramique découvert dans les puits des chantiers des Longrais par B. Edeine en 1966 et 1967 (DAO, C. Marcigny, Inrap).

Figure 7: Ceramics found in Les Longrais pits by B. Edeine in 1966 and 1967.

\section{La stratigraphie « naturelle » du terrain}

La zone concernée par le diagnostic de 2008 est implantée sur le plateau, dans l'axe de l'éperon du Mont Joly, le long d'une barre de grès/quartzite (fig. 8), au contact entre le Bassin parisien et les premiers renforts du Massif armoricain. Le site se trouve ainsi dans une situation géologique complexe, entre les roches du massif ancien au sud formant une élévation de quelques mètres, des nappes d'argile (emballant grès, quartzite et silex) dans le tiers sud de la zone explorée et un substrat calcaire quasiaffleurant mais lardé de poches d'argile à silex (poche de décalcification) sur le reste du terrain.

Une approche précise de la stratigraphie «naturelle» de ce secteur a donc été menée en parallèle à la fouille de manière à bien caractériser les conditions d'implantation des structures (fig. 8). Cette approche a de plus été complétée par des tests réalisés sur les matières siliceuses (prélèvement de blocs naturels, taille expérimentale), de manière à caractériser la qualité des silex présents dans le sous-sol des Longrais. Au terme de cette étude, il est possible de présenter dans ses grandes lignes la stratigraphie générale du site.

La barre de grès et quartzite n'a pas été sondée mais elle est caractérisée par un chaos rocheux où pointent un grand nombre de blocs de grand module. Les dalles de quartzite sont très fréquentes et c'est probablement deux d'entre elles que B. Edeine à redressées dans les années 60 , pensant qu'il s'agissait de menhirs renversés. 
Le long de la barre, une nappe d'argile, emballant de nombreux cailloux de grès, quartzite et silex, se développe sur des surfaces variables. L'absence de sondage profond dans ce secteur ne nous permet pas de connaître la puissance de cet horizon qui dépasse $0,80 \mathrm{~m}$ de profondeur sous la terre végétale, épaisse d'une vingtaine de centimètre sur l'ensemble de la zone explorée. Dans cette couche, le silex, très gélifracté et généralement concassé, est bien entendu inexploitable.



Figure 8 : Vue en plan des différents horizons rencontrés lors des décapages et implantation des principales stratigraphies du site, selon un axe sud-nord (A-B) : coupes T4, 52, T6, 44, 10, 70, 2 et 83 (DAO, C. Marcigny, Inrap).

Figure 8: Plan of the main horizons encountered while scraping the site and location of the main stratigraphies.

Plus vers le nord-est, le substrat calcaire (bathonien) est directement accessible sous une couche de limon gris de 0,15 à 0,50 m d'épaisseur, elle-même sous-jacente à la terre végétale. Le calcaire est pulvérulent et contient par passées des rognons de silex de qualité très inégale. Dans un cas (coupe 44 , fig. 8 ), le silex se présente sous la forme d'un véritable banc très compact d'une vingtaine de centimètres d'épaisseur; ce silex, de couleur grise, est gélifracté et d'une qualité trop médiocre pour la taille. Sur cette même coupe, à près de deux mètres de profondeur, un nouveau niveau à silex de même couleur est observé, mais cette fois-ci apte à la taille (silex gris foncé sursilicifié à cortex fin et lisse d'aspect légèrement satiné, comparable au silex dit « du Cinglais »).

Le substrat présente ponctuellement des cuvettes de dissolution qui se repèrent en plan par un niveau d'argile rouge formant des taches plus ou moins circulaires de 4 à $6 \mathrm{~m}$ de diamètre (fig. 8). Ces cuvettes peuvent atteindre $3 \mathrm{~m}$ de profondeur. Elles présentent dans leurs remplissages des niveaux de silex gris surcilicifié de très bonne qualité, comme sur la coupe 10 de la figure 8 . Cette stratigraphie générale est bien entendu sujette à des variations sur l'ensemble de la surface diagnostiquée. 


\section{La minière}

19 En conformité avec les observations faites par B. Edeine dans les années 1960, le site s'est révélé riche en structures ; parmi celles-ci les puits de minière à silex dominent très largement. 38 puits ont ainsi été plus ou moins partiellement dégagés en surface lors de la réalisation des tranchées de diagnostic, dont 9 ont été fouillées à 50 \% (fig. 9).

Devant l'importance des vestiges découverts, le Service régional de l'Archéologie a prescrit une fouille à l'emplacement des constructions projetées. Cette fouille n'aura malheureusement pas lieu suite à la modification substantielle du projet d'aménagement. Les résultats présentés ici sont donc les seuls disponibles, sans doute pour longtemps puisque les terrains concernés ont désormais réputés non constructibles. Les informations acquises durant les quelques jours du diagnostic paraissent toutefois suffisamment importantes pour faire l'objet d'un article présentant les données brutes puits par puits.

\section{Le puits 1 de la tranchée T 1}

21 Le puits 1 est creusé à l'emplacement d'une cuvette de dissolution du calcaire bathonien (fig. 10). Il s'agit en fait d'une très large fosse de presque $4 \mathrm{~m}$ de diamètre qui descend à un peu plus de $3 \mathrm{~m}$ de profondeur pour atteindre le niveau à silex. Après extraction, la cuvette a été comblée avec les terres issues de son creusement. On y retrouve ainsi une alternance de couches de limon plus ou moins argileux, ponctuées de bloc de silex rejetés car de qualité trop médiocre. Le sommet de la fosse a livré de nombreux déchets de taille, ultimes témoins des activités qui se sont déroulées autour du puits. Cette structure est celle qui a livré le plus de vestiges parmi toutes celles sondées à la fouille. Dans le cadre de la description des autres lots mobiliers, c'est elle qui nous servira de référence.

22 Le mobilier lithique issu du remplissage de ce puits 1 se compose de 280 artefacts de plus de $2 \mathrm{~cm}$ de long et de 276 esquilles, pour un poids de $54420 \mathrm{~g}$, dont 7 blocs de matière première plus ou moins mis en forme, pour un poids de $47260 \mathrm{~g}$ (tabl.1). Le mobilier est issu des couches intermédiaires du remplissage, dans les niveaux limoneux. Il apparaît à quelques centimètres sous le labour, vers $0,40 \mathrm{~m}$ de profondeur sous le sol actuel; il est plus dense entre 0,50 et $0,60 \mathrm{~m}$ puis de moins en moins abondant jusqu'à $1,20 \mathrm{~m}$. Le mobilier a été ramassé manuellement lors de la fouille des concentrations de silex entre chaque passe de pelle mécanique. Cela explique en particulier le nombre important d'esquilles mises au jour.

23 Le matériau concerné est exclusivement le silex bathonien gris local. Il est présent soit sous sa forme sursilicifiée de très bonne qualité (grande majorité des artefacts), soit sous sa forme désilicifiée et très grenue (quelques éclats d'initialisation, quelques gros blocs).

\section{Débitage}

La série se partage entre $15 \%$ d'éclats très corticaux (qui participent de l'initialisation des blocs), $28 \%$ d'éclats divers, $25 \%$ d'éclats larges et fins (probablement tirés dans le cadre du façonnage des flancs des blocs et nucléus pour l'établissement des crêtes latérales, ou encore pour le façonnage d'outils tels que des tranchets), $9 \%$ de produits 
allongés (lames et lamelles, entières ou fragmentées), $10 \%$ de produits d'entretien des nucléus (crête, avivage, flanc); on note enfin $1,4 \%$ de nucléus à éclats et $0,7 \%$ de nucléus laminaires.

Les supports laminaires - lames et lamelles - présentent les caractéristiques de plusieurs types de production. Les plus évidentes concernent des produits réguliers, à 3 pans et d'épaisseur constante. Le talon est large, avec une concavité résultant de l'enlèvement d'une esquille d'avivage. Le bulbe est bombé et court. Ces stigmates indiquent sans conteste l'usage de la percussion indirecte, ce qui est corroboré par deux nucléus à lames, fragmentaires mais présentant des négatifs d'enlèvements laminaires très réguliers et un esquillement rasant du plan de frappe. Quelques autres supports laminaires portent des traces moins évidentes ; ces supports sont également moins réguliers. La percussion directe tendre est proposée mais, devant la majorité de produits témoignant de la percussion indirecte, il convient de rester prudent.

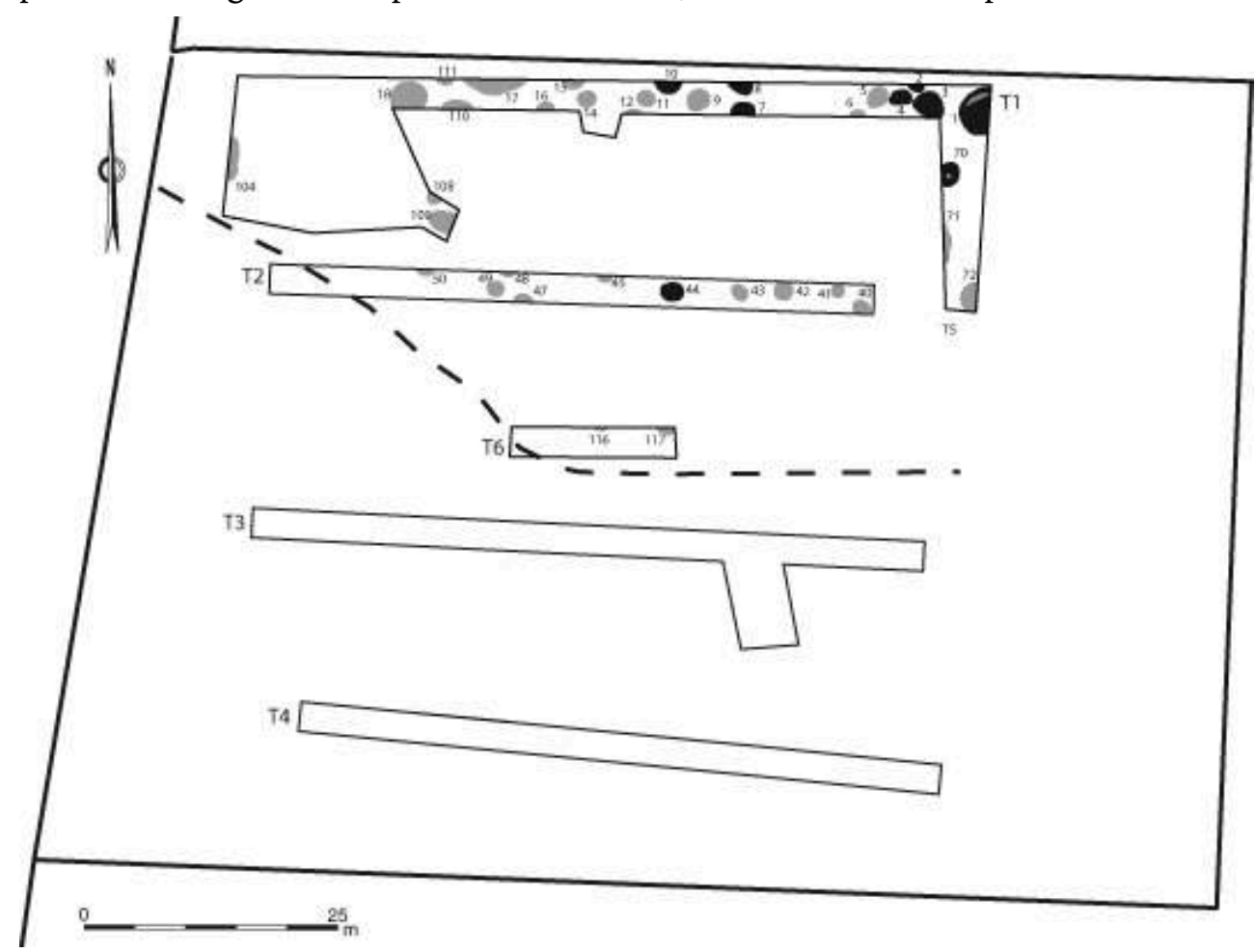

Figure 9 : Implantation des puits de mine ; en grisé, les puits simplement repérés, en noir, les puits fouillés et en tireté l'extension hypothétique de la minière (DAO, C. Marcigny, Inrap).

Figure 9: Location of mine pits; in grey, identified; in black, excavated. Dotted line marks hypothetical limit of the mining area. 


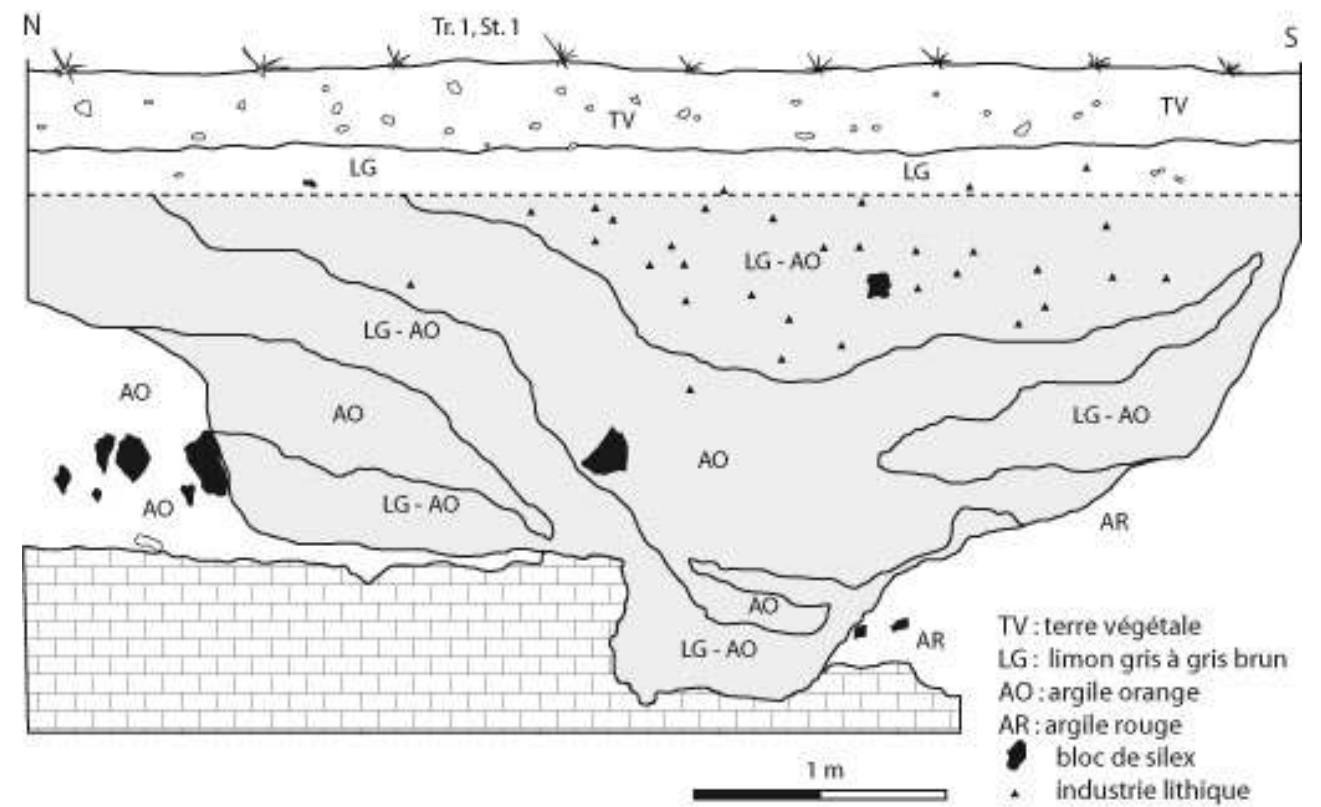

Figure 10 : Coupe du puits 1 de la tranchée 1 (DAO, E. Ghesquière, Inrap).

Figure 10: Section of pit 7 in trench 1.

Les produits d'entretien des nucléus - lames à crête, tablettes ou éclats d'avivage, flancs de nucléus à enlèvements lamellaires - sont présents à hauteur de $9 \%$ dans la série. Tous se rattachent à un débitage de produits très réguliers. Les lames à crête portent les stigmates déjà décrits d'un débitage par percussion indirecte. Les tablettes d'avivage portent sur leur face supérieure les négatifs d'esquilles caractéristiques. L'épaisseur de ces tablettes varie de quelques millimètres à $1,5 \mathrm{~cm}$.

Un gros bloc de silex initialisé a été découvert dans la structure 1. Il mesure $28 \mathrm{~cm}$ de haut et 12 de large. De forme ogivale, son façonnage s'est arrêté à un stade encore grossier. Il présente toutefois l'amorce de deux crêtes latérales. 


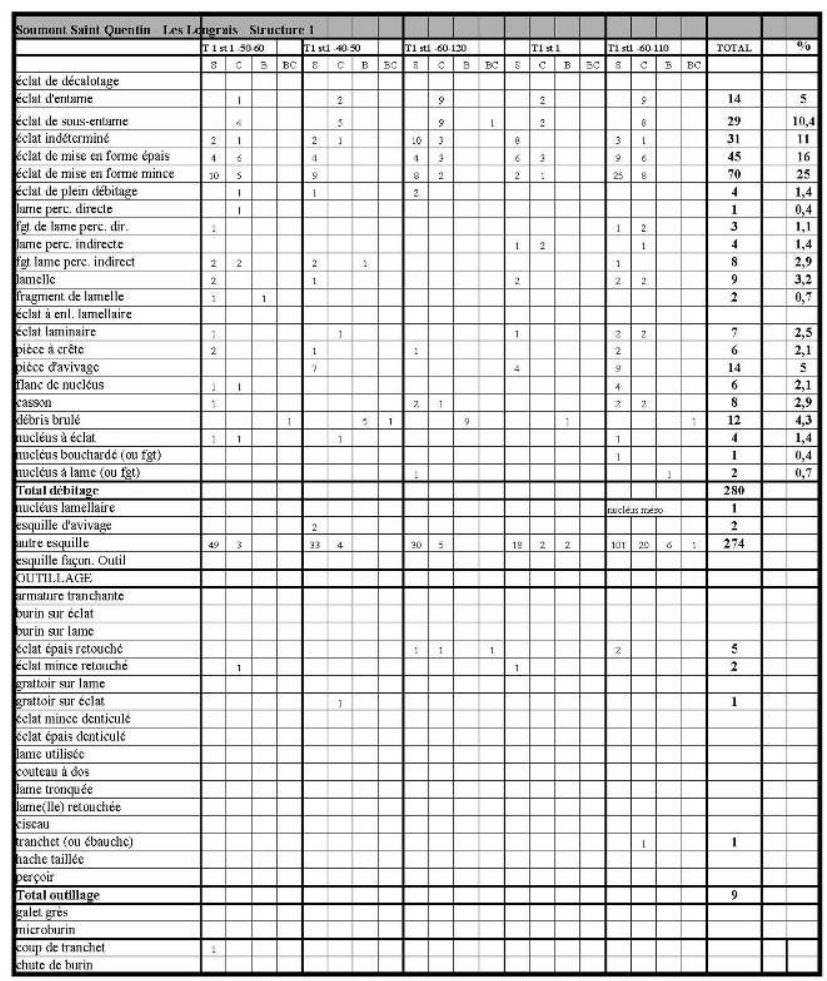

Tableau 1 : Inventaire du mobilier lithique du puits 1.

Table 1: Inventory of lithic assemblage from pit 1 .

\section{Outillage}

Il se réduit à 11 pièces, soit $3 \%$ du corpus.

- Un grattoir sur éclat assez large présente une retouche semi-abrupte débordante du front.

- Un tranchet présente une morphologie trapézoïdale et une taille réduite (fig. $11, \mathrm{n}^{\circ} 2$; $\mathrm{L}_{.}=6,5 \mathrm{~cm}$ ). Les bords présentent une retouche abrupte ; l'extrémité n'a pas fait l'objet d'un enlèvement « coup de tranchet ». Dans un premier temps considérée comme une ébauche, cette pièce a cependant montré sur ses bords de probables traces d'emmanchement (étude tracéologique en cours par F. Charraud).

- Cinq éclats de grandes dimensions présentent des enlèvements profonds créant une retouche écailleuse régulière. Il ne s'agit vraisemblablement pas d'une retouche intentionnelle, mais d'enlèvements consécutifs à une utilisation.

- Deux pièces esquillées ont également été reconnues dans cette structure (fig. $11, \mathrm{n}^{\circ} 4$ et 5 ). Elles évoquent un travail en percussion directe ou indirecte sur une matière animale dure (os ou bois de cervidé).

- Deux petits éclats minces portant une retouche régulière complètent l'assemblage.

\section{Comparaisons et attribution chrono-culturelle}

La matière utilisée est un silex bathonien sursilicifié tel qu'on le trouve dans les placages d'argile à silex de la Plaine de Caen. Il est surnommé "silex du Cinglais », en référence au gisement du plateau des Moutiers-en-Cinglais (Calvados), exploré par J. Desloges en 1988 (Desloges et Ghesquière, 2007). Ce matériau se rencontre sur la plus vaste plaque d'argile à silex de la Plaine de Caen, selon les travaux universitaires de $\mathrm{S}$. Coutard (1998, repris dans Ghesquière et Marcigny, 1998). Cette commune est aussi le 
lieu du plus grand atelier de production de lames découvert dans la Plaine (Desloges et Ghesquière, 2007). L'usage de ce silex d'argile a pour l'instant été identifié depuis le Rubané final $\mathrm{du}$ Bassin Parisien/Bliquy- Villeneuve-Saint-Germain ancien (Colombelles: Ghesquière et Marcigny, 2000; Billard et al., 2004), jusqu'au BVSG (Mondeville «Haut-Saint-Martin»: Chancerel et Ghesquière, 2006) et même au Néolithique moyen I (Ernes et Condé-sur-Ifs : Dron et San Juan, 1992).

Le débitage est orienté au moins en majorité vers la production de lames régulières par percussion indirecte (même si une utilisation des gros éclats d'épannelage pour la réalisation de tranchets n'est certainement pas négligeable). Dans la région, ce type de production est caractéristique du début du Néolithique. Elle y correspond plus précisément à une utilisation de ces supports réguliers pour la réalisation de burins, armatures de faucilles et armatures de flèches. Ce type de débitage a été mis en évidence dans la région au RFBP/BVSG ancien sur le site de Colombelles "Lazzaro ", sur les sites BVSG de Fontenay-le-Marmion « La Grande pièce » (Giraud et Juhel, 2004) et Mondeville «HSM»; au Cerny, on le retrouve à Condé-sur-Ifs (Ghesquière et Marcigny, 1998 ; inédit, fouille J.-L. Dron). Sous la forme de produits finis (lames régulières transformées en outils), on retrouve ces outils dispersés en Basse-Normandie et en Bretagne, dans les mêmes contextes chronologiques.

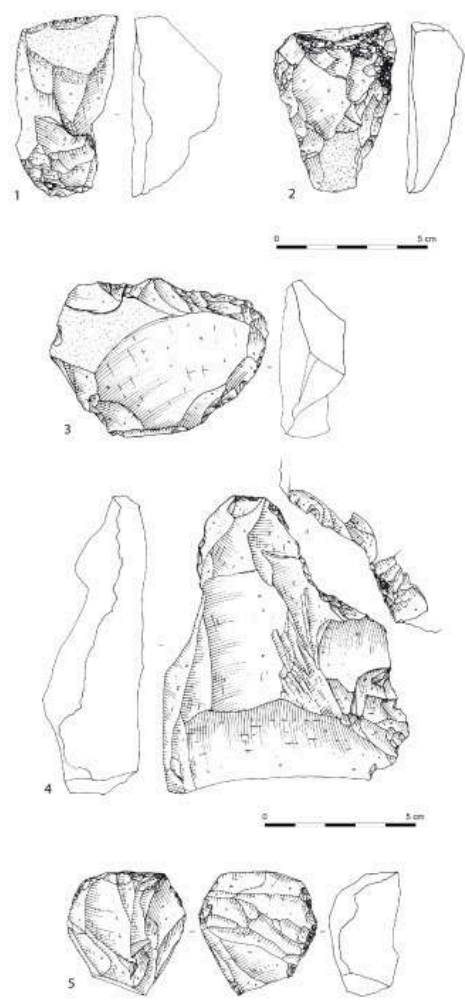

Figure 11 : Mobilier de la structure 1 ; outillage commun et pièces esquillés (dessin F. Charraud, Inrap). Figure 11: Assemblage from structure 1: common tools and splintered pieces.

31 Aucun outil caractéristique n'a été mis en évidence dans le puits 1. Les gros éclats retouchés pourraient trouver des équivalents dans la série des Moutiers-en-Cinglais (Desloges, rapport inédit ; Ghesquière, étude inédite), comme dans la série de la minière de Ri, plus récente puisque du Néolithique moyen II (Marcigny et al., 2007). Leur hypothèse d'usage dans un cadre minier est avancée ici comme pour les deux autres 
sites (affûtage des outils de creusement ?). De tels outils semblent en effet avoir peu de place dans un contexte domestique si on les compare à ceux des habitats déjà cités.

Le tranchet trouve sa place dans un contexte chronoculturel beaucoup plus large en Basse-Normandie. Son apparition est attribuée dans la région au BVSG moyen (Tilly-laCampagne : Giraud, étude en cours) et au BVSG «à cordon et tranchet » (Mondeville «HSM »), même si de telles pièces restent rares dans la région. La Haute-Normandie est beaucoup plus riche en ce type de pièce pour la même période (Saint-Vigord'Ymonville: Marcigny et al., 2002 ; Guichainville "Long Buisson »: Carpentier et al., 2005). En Basse-Normandie, les tranchets se rencontrent beaucoup plus nombreux dans le Néolithique moyen. On ne peut que rapprocher l'exemplaire du puits 1 de l'abondant effectif issus de ramassages de surface sur le site des Longrais.

$\mathrm{Au}$ regard des différentes informations rassemblées (matière première, production laminaire indirecte, présence de tranchet), il semble que l'on puisse resserrer la chronologie de ce remplissage sur le BVSG et le Cerny, dans une fourchette probable de 5000 à 4500 av. J.-C.

\section{Les autres puits de tranchée 1}

\section{Les puits 2,3 et 4}

Les puits 2, 3 et 4 ont été fouillés ensemble lors d'un même sondage réalisé au godet de $3 \mathrm{~m}$. Ce sondage avait pour principal objet, au-delà du relevé de coupes stratigraphiques, d'identifier d'éventuels recoupements entre puits témoignant d'une exploitation du site sur la longue durée. La fouille a montré que les puits, même s'ils n'étaient pas obligatoirement synchrones, ne se recoupaient pas (fig. 12).

Les trois puits se présentent sous la forme de cuvettes peu profondes (de l'ordre d'un mètre sous le décapage). Ils montrent, au niveau de la couche à silex, des diverticules permettant d'exploiter celle-ci au maximum en évitant la manipulation trop importante de stériles. Leurs comblements sont, comme pour le puits 1, constitués des déblais (ici une argile orange à rouge avec des passées de limon gris).

La structure 2 a livré 18 artefacts de longueur supérieure à $2 \mathrm{~cm}$ et quatre esquilles, inclus dans les couches de remplissage limoneux. La matière utilisée est le silex bathonien gris local. Malgré le faible nombre de vestiges, on note deux lames présentant les stigmates du débitage par percussion indirecte. Il semble que l'on puisse considérer ce mobilier comme globalement comparable (matière première, débitage) à celui mis en évidence dans la structure St. 1.

\section{Les puits 7 et 8}

Ces deux puits sont profonds de 1,20 à $1,50 \mathrm{~m}$ sous le décapage ( $2 \mathrm{~m}$ sous le sol naturel moderne) et sont reliés ensemble par une très courte galerie (fig. 13). Leurs profils sont en tronc de cône avec une ouverture à la surface très légèrement évasée. Au niveau de la couche à silex (ici encore de très bonne qualité), les puits s'évasent, formant des diverticules voire de petites galeries (dont l'une n'était même pas remplie de sédiment). Leur comblement comporte à la base un épais remblai de calcaire pulvérulent, surmonté de blocs de silex abandonnés, puis viennent des couches de limon brun à brun gris parfois ponctué de calcaire. Ces types de profil et de comblement sont en tout point semblables à ce que l'on connaît sur d'autres minières régionales, en particulier à 
Ri où le calcaire se présente sous la même forme pulvérulente (Marcigny et al., 2007). Ces deux puits n'ont pas livré de mobilier.

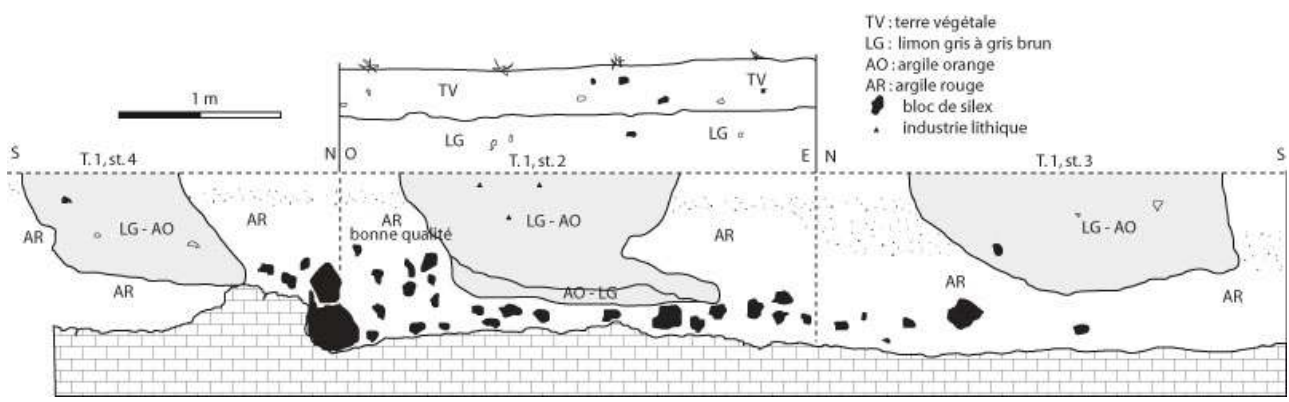

Figure 12 : Coupes des puits 4,2 et 3 de la tranchée 1 (DAO, E. Ghesquière, Inrap).

Figure 12: Sections of pits 4, 2 and 3 in trench 7.

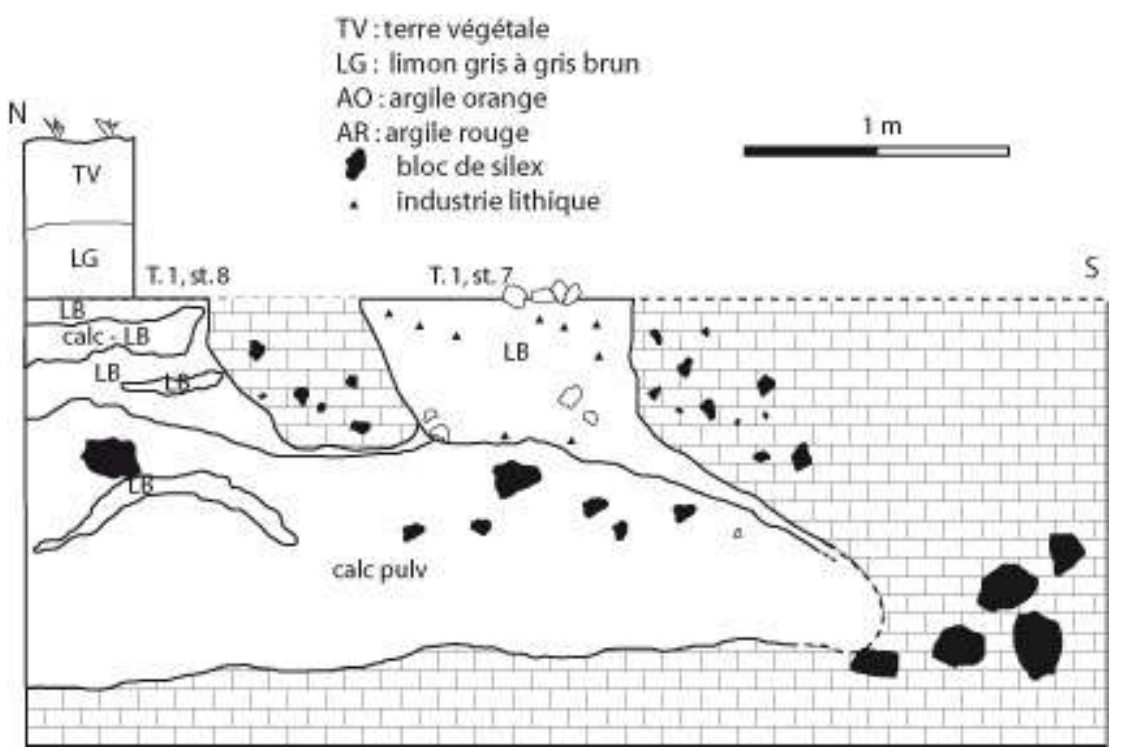

Figure 13 : Coupes des puits 7 et 8 de la tranchée 1 (DAO, E. Ghesquière, Inrap).

Figure 13: Sections of pits 7 and 8 in trench 1.

\section{Le puits 10}

Ce puits a des parois presque droites (mais évasées au niveau de l'ouverture) et un fond plat à près de deux mètres de profondeur sous le décapage: (fig. 14). Au fond de l'excavation, le puits présente un très court diverticule dans le calcaire, peut être à la recherche d'une couche de silex qui ici n'existe pas. Dans l'argile, le creusement a atteint le niveau à silex mais ne l'a visiblement pas exploité alors qu'il semble de très bonne qualité. Nous avons peut-être à faire dans ce cas à un "puits test » qui n'avait pour vocation que d'identifier le potentiel de la couche à silex. La présence de plusieurs autres puits (St. 11, 12,14, 15 et 16) dans le même secteur est peut-être le témoin de l'intensité de l'exploitation de la cuvette de dissolution après détection de la profondeur des silex. Ou alors, dans un autre scénario chronologique, St. 10 pourrait correspondre à une volonté d'extension de la minière vers un secteur plus calcaire, extension abandonnée après ce test qui aurait été jugé décevant.

Le mobilier lithique est issu du remplissage supérieur limoneux. Il se compose de 25 artefacts d'une longueur supérieure à $2 \mathrm{~cm}$ (parmi lesquels 4 outils), et de 19 esquilles. Le matériau employé est exclusivement le silex bathonien gris local, soit sous sa forme 
sursilicifiée de très bonne qualité (grande majorité des artefacts), soit sous sa forme désilicifiée et très grenue (quelques éclats).

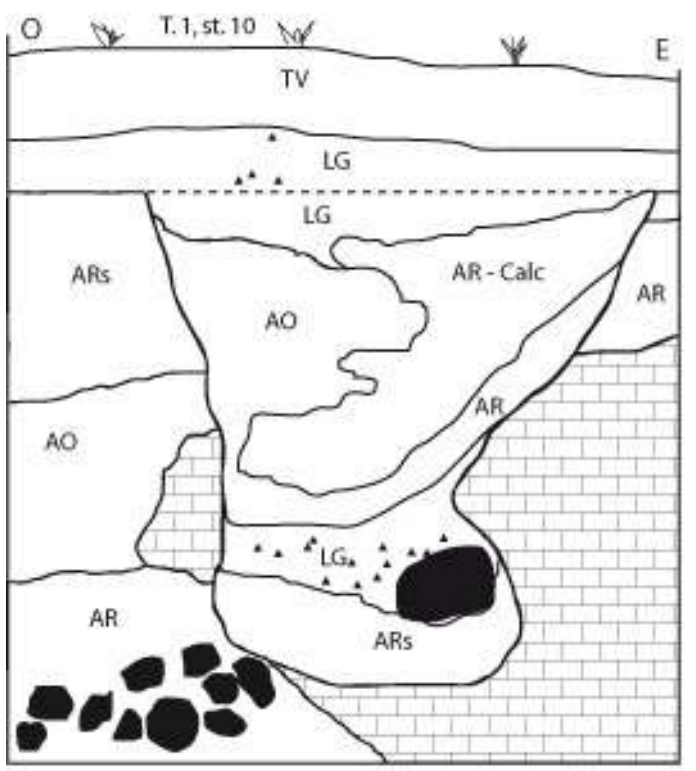

$$
\begin{aligned}
& \text { TV:terre vigutale } \\
& \text { LG: limon gris à gris brun } \\
& \text { AO:arglle crange } \\
& \text { AR:argile rouge } \\
& \text { bloc de silex en place } \\
& \text { industrie lithique }
\end{aligned}
$$

Figure 14 : Coupe du puits. 10 de la tranchée 1 (DAO, E. Ghesquière, Inrap).

Figure 14: Section of pit 10 in trench 7.

Débitage et outillage. L'ensemble des vestiges retrouvés se rapporte à un débitage laminaire par percussion indirecte. Deux nucléus à enlèvements réguliers sont présents, de même que deux flancs de nucléus à négatifs d'enlèvements laminaires et huit éclats très minces de mise en forme (crêtes ou façonnage d'outils épais). Aucune lame entière ou fragmentée n'est toutefois à rapporter dans la série. La présence d'un nucléus à éclat suppose toutefois une production, même marginale de supports courts. L'outillage se compose d'un éclat mince retouché, d'un éclat épais denticulé et de deux ébauches de tranchet sur gros éclats non corticaux, présentant des enlèvements discontinus directs et inverses.

Comparaisons et attribution chrono-culturelle. La matière utilisée dans le cadre du débitage est un silex bathonien sursilicifié, comme celui exploité sur le site. Le débitage est orienté vers la production de lames régulières par percussion indirecte; un débitage d'éclat (marginal ?) est toutefois à signaler à travers la présence d'un nucléus. L'outillage (éclat épais denticulé, ébauches de tranchet) est comparable à celui des autres lots du site. Il semble que l'on puisse attribuer le mobilier à la même fourchette chronologique que celui de la structure 1, soit entre le BVSG et le Cerny.

\section{Le puits 11}

La structure 11 n'a pas été fouillée. Son décapage de surface a toutefois livré cinq artefacts supérieurs à $2 \mathrm{~cm}$ et deux esquilles. La matière première est le silex bathonien gris exploité sur le site. 

particulièrement dans le RFBP/BVSG ancien de Colombelles (Ghesquière et Marcigny, 2000). Au BVSG, ce type d'outil est rare dans les séries (très peu étoffées il est vrai) de Basse-Normandie. En revanche, on le retrouve au Cerny ancien (Condé-sur-Ifs: Ghesquière et Marcigny 1998).

Au regard des différents éléments rassemblés (matière première, nucléus laminaires, tranchet), il semble que l'on puisse attribuer le mobilier de ce puits 14 à la même fourchette chronologique que l'assemblage de la structure 1. La présence d'un grattoir sur lame (mais est-elle représentative ?) est toutefois peu fréquente en contexte BVSG, 
du moins par rapport aux connaissances encore limitées que nous avons de cette période dans la région.
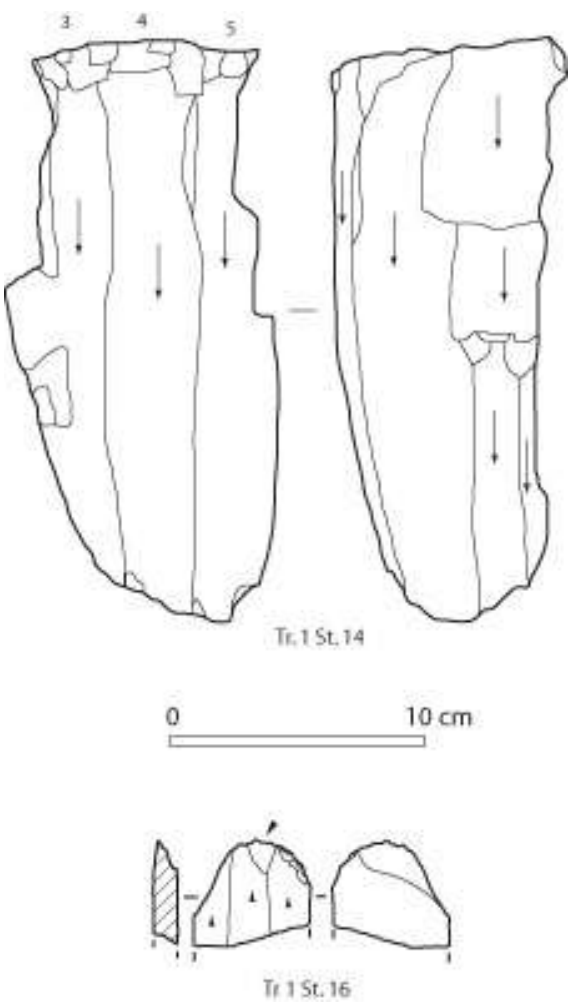

Figure 15 : Mobilier lithique retrouvé dans les puits 14 et 16 (DAO, E. Ghesquière, Inrap).

Figure 15: Lithic assemblage from pits 14 and 16.

\section{Le puits 16}

La structure, non fouillée, a livré lors de son nettoyage de surface trois artefacts de plus de $2 \mathrm{~cm}$ et une esquille. La matière première est toujours le silex bathonien gris local.

Malgré le faible nombre des vestiges, on y note un fragment de nucléus laminaire qui présente les caractéristiques générales d'une production régulière par percussion indirecte, une ébauche de tranchet sur gros éclat cortical et un microburin sur lame à trois pans très régulière (fig. 15$)$. Cette pièce, de très grand module ( $23 \mathrm{~mm}$ de large) est peut-être le déchet de fabrication d'une armature de faucille ou de flèche.

Il semble que l'on puisse là encore considérer ce mobilier comme globalement comparable (matière première, débitage) à celui mis en évidence dans la structure 1 et le placer dans la même fourchette chronologique. On note que la présence de microburin est très rare en contexte domestique dans la région (une pièce de même module à Mondeville «HSM »), même si des armatures de flèches réalisés par cette méthode de fracturation ont été découvertes à Fontenay-le-Marmion «La Grande Pièce » (Giraud et Juhel, 2004).

\section{Les puits 17 et 18}

La structure 17, également non fouillée, a livré lors de son décapage cinq artefacts d'une longueur supérieure à $2 \mathrm{~cm}$. La matière première en est toujours le silex bathonien gris local. Malgré le faible nombre de vestiges, deux éclats de flancs de 
nucléus présentant des négatifs d'enlèvements laminaires réguliers suggèrent un débitage par percussion indirecte. Leur présence renvoie globalement à la même fourchette chronologique que celle proposée pour la structure 1 .

La structure 18 n'a pas non plus été fouillée; elle a livré, lors de son nettoyage de surface, cinq artefacts supérieurs à $2 \mathrm{~cm}$ et deux esquilles. La matière première est toujours le silex bathonien gris local. Malgré le faible nombre de vestiges, on note que les deux éclats de mise en forme minces sont tout à fait comparables à leurs homologues de la structure 1. Il semble que l'on puisse là encore considérer ce mobilier comme lui étant globalement comparable (matière première et débitage).

\section{Le puits 44 de la tranchée 2}

Le puits 44 est la seule structure d'extraction fouillée dans la tranchée 2. La minière nous paraissait en effet suffisamment documentée au niveau de la phase diagnostic et il a été décidé de limiter les excavations profondes dans ce secteur, le laissant en réserve pour une éventuelle fouille à venir. Toutefois, la structure 44 apparaissait en surface comme une fosse de deux mètres de diamètre, comblée de limon gris et inscrite dans un plaquage d'argile rouge, proche en texture de la nappe de grès et de quartzite que l'on rencontre quelques mètres plus au sud. Le contexte géologique étant différent de ce que l'on connaissait dans la tranchée 1, entre le calcaire bathonien presque affleurant et ses cuvettes de dissolution, il nous a semblé opportun d'échantillonner ce nouveau puits.

En coupe (fig. 16), le puits adopte le profil d'un tronc de cône très évasé. En effet, après avoir traversé un véritable banc de silex très compact mais impropre à la taille, le fond de l'excavation utilise ce banc comme plafond et file en diverticules sur au moins deux mètres de long de manière à exploiter à sa base la couche de silex de bonne qualité. Ces diverticules se développent ainsi tout autour du puits formant une extraction en "pétales de fleur ».

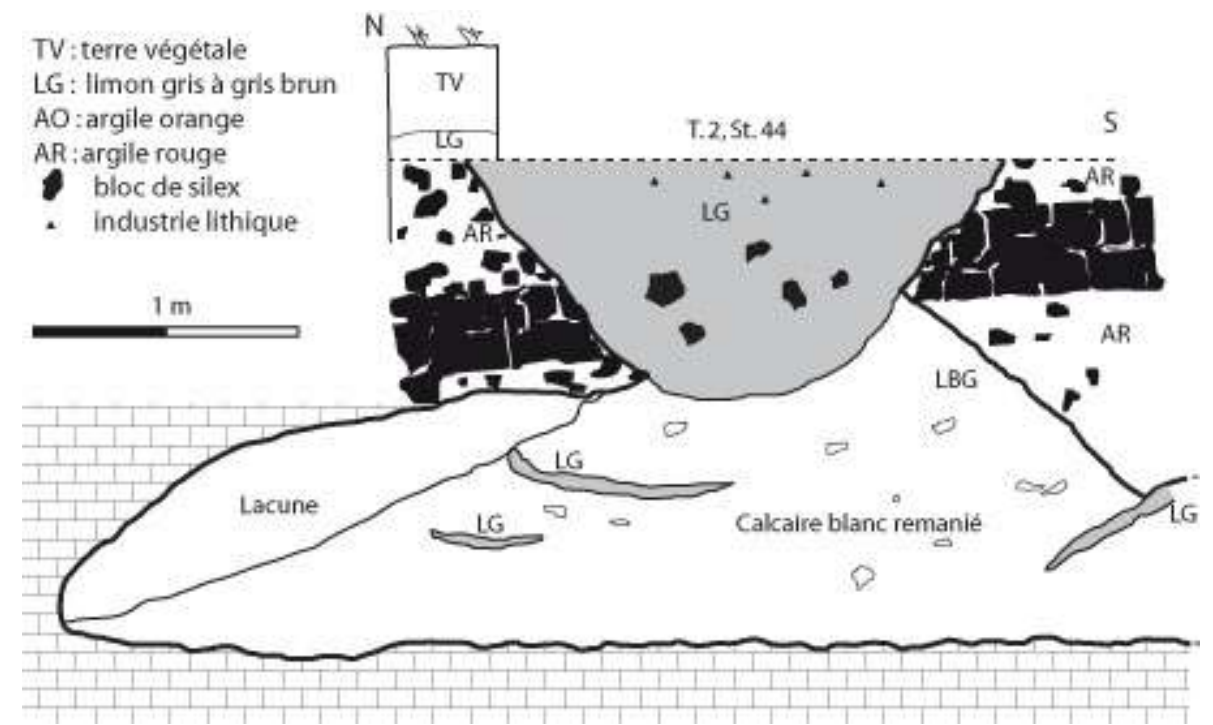

Figure 16 : Coupe du puits 44 de la tranchée 2 (DAO, E. Ghesquière, Inrap).

Figure 16: Section of pit 44 in trench 2. 
remplissage du puits est presque exclusivement constitué de calcaire pulvérulent à l'exception de son accès comblé de limon gris. Les diverticules quant à eux présentent un comblement de même nature mais extrêmement lacunaire.

61 Le mobilier lithique issu du remplissage se compose de 15 artefacts longs de plus de $2 \mathrm{~cm}$ (parmi lesquels 6 outils) et de 6 esquilles, pour un poids de $4340 \mathrm{~g}$. Ce mobilier est issu de la couche supérieure du remplissage limoneux du puits. Il est exclusivement en silex bathonien gris local.

Débitage. La série, très limitée, ne permet une approche que sous forme de présence/ absence des pièces. Les deux éléments les plus pertinents sont deux nucléus laminaires arrivés en fin de comblement, qui portent des stigmates caractérisant le débitage laminaire indirect : régularité des négatifs d'enlèvement laminaires, esquillement du plan de frappe... La présence de deux fragments de lame à crête, produits très probablement par percussion indirecte (régularité de l'épaisseur), semble confirmer ce diagnostic. Un nucléus à éclats présente quelques enlèvements à la surface d'un bloc de silex encore largement cortical. Malgré une fouille manuelle partielle, très peu d'esquilles sont issues du niveau de «concentration » des silex. On note par ailleurs la disproportion entre la présence des deux nucléus réguliers et la poignée d'autres déchets de taille.

Outillage. Il concerne 3 pièces seulement.

- Un éclat épais retouché et un autre, denticulé, portent une retouche écailleuse profonde. Ces deux pièces massives rappellent les cinq éclats comparables de la structure 1.

- Une ébauche de tranchet bifaciale est très probable. La retouche directe et inverse est très partielle sur ce gros éclat de sous-entame mais la morphologie générale de la pièce suggère bien une ébauche de tranchet.

Comparaisons et attribution chrono-culturelle. La matière utilisée est le même silex bathonien sursilicifié gris que dans les autres puits fouillés. Le débitage est orienté, au moins en majorité, vers la production de lames régulières par percussion indirecte. Une production ponctuelle d'éclats de plein débitage (faible nombre d'éclats par nucléus) et une utilisation de gros éclats d'épannelage pour la réalisation d'outils bifaciaux sont également présentes. Le débitage coïncide avec celui observé dans la structure 1 . Il renvoie à la même fourchette chronologique. Aucun outil caractéristique n'a été mis en évidence. La probable ébauche de tranchet renvoie aussi aux conclusions proposées pour la Structure 1.

\section{Le puits 70 de la tranchée 5}

La structure 70 a été fouillée car, lors de son décapage de surface, la présence d'un petit niveau charbonneux placé en son centre détonait par rapport aux structures environnantes. Dans l'idée de fouiller une structure d'un nouveau type (peut-être à vocation domestique), il a été décidé d'y effectuer une coupe.

Après fouille, la structure peut, une fois de plus, être considérée comme un puits, éventuellement avorté si on la compare à St. 10 de la tranchée 1. Descendant à un peu plus de deux mètres sous le niveau de décapage, l'excavation présente des parois droites et un fond plat, Elle s'arrête au contact de la couche de silex, ici de très mauvaise qualité, ce qui a mis un terme à son creusement. 

trois esquilles dans sa couche supérieure. La matière première est toujours le silex bathonien gris local. Malgré le faible nombre de vestiges, on note que les deux éclats de mise en forme minces sont tout à fait comparables à leurs homologues de la structure 1 (crête, façonnage d'outils...). Il semble que l'on puisse considérer ce mobilier comme globalement comparable.

\section{Conclusions}

Les données acquises à l'issue de cette évaluation archéologique sont nombreuses et présentent un indéniable intérêt scientifique. La confirmation d'une zone d'extraction, déjà pressentie par B. Edeine dans les années soixante, et qui s'est avérée particulièrement dense, est un des apports majeurs. Dans le cadre du diagnostic, près d'une quarantaine de puits ont pu être examinés en surface et neuf ont été fouillés à $50 \%$.

69 Cette minière avait pour fonction l'exploitation du silex bathonien, inclus soit dans les argiles de recouvrement du site (dans des cuvettes de dissolution ou en nappe), soit dans le calcaire sous-jacent (entre $1 \mathrm{~m}$ et $1,50 \mathrm{~m}$ de profondeur sous le sol actuel). Le débitage était réalisé in situ, comme le suggère la présence de l'intégralité de la chaîne de production dans le comblement des puits (extraction, blocs bruts, blocs mis en forme, éclats d'initialisation, éclats de mise en forme, éclats d'entretien, lames, nucléus, esquilles). Il vise à la production par percussion indirecte de lames régulières, ainsi qu'à celle d'éclats courts (production marginale) et au façonnage de tranchets bitronqués ou bifaciaux.

70 L'outillage est très discret et ne semble pas correspondre à un assemblage domestique. Il consiste principalement en gros éclats retouchés ou denticulés, pour lesquels un rôle dans le système d'exploitation n'est pas exclu (affûtage des outils en bois ?). Le reste de l'outillage, à quelques exceptions près, consiste en ébauches de tranchet. La présence de trois grattoirs et d'un microburin reste anecdotique.

71 L'ensemble évoque indiscutablement le Néolithique ancien ou le Néolithique moyen I, ce qui ferait des Longrais une des minières attestées parmi les plus anciennes d'Europe, rejoignant celles de Pologne (Lech, 1982), d'Italie (Galiberti et al., 1998) et d'Espagne (Capote et al., 2008) et l'une des seules sondées pour cette époque en France

Depuis vingt ans, les fouilles de minières, réalisées au hasard des grands travaux d'aménagement, ont apporté leur lot d'informations pratiques, sur les techniques d'extraction par exemple, mais les modèles d'exploitation communément admis demeurent limités. L'étude des minières de silex et de leur raison d'être sur la durée ne peut se concevoir que dans le cadre d'un investissement régional de longue haleine, combinant la fouille de sites d'extraction et d'ateliers mais aussi l'identification des produits et de leur diffusion. C'est pourquoi le diagnostic des Longrais revêt une importance toute particulière dans le contexte régional. Il n'est plus temps de valider les informations qui nous sont parvenues des fouilles de 1966, mais il s'agit désormais de répondre à un questionnement précis, émergé entre-temps. S'il est confirmé que la production des Longrais concerne essentiellement des tranchets et des lames dans une ambiance culturelle marquée par des éléments domestiques BVSG ou Cerny, il s'agirait, comme souligné plus haut, des extractions de profondeur les plus anciennes connues à 
ce jour en France; elles seraient contemporaines de la vague pionnière de néolithisation et par conséquent bien antérieures au phénomène minier "normatif ". Cette exploitation, dont il faudrait déterminer les signatures, serait peu ou prou contemporaine de l'exploitation des rognons de l'argile à silex dit «du Cinglais». Rappelons que celui-ci constitue l'une des ressources principales du Néolithique ancien pour la production de lames en Normandie et en Bretagne. Ainsi deux stratégies d'acquisition que l'on oppose d'ordinaire pourraient avoir coexisté au Néolithique ancien : le système minier d'une part et le traditionnel ramassage des rognons dans les biefs à silex d'autre part.

\section{BIBLIOGRAPHIE}

Aubouin, J. et Labesse, B., 1985 - Nouvel atlas des formes du relief, Paris, Nathan.

Bailloud, G., 1964 - Le Néolithique dans le Bassin Parisien, Paris, CNRS ( $2^{\mathrm{e}}$ supplément à Gallia Préhistoire ; seconde édition mise à jour 1972), $433 \mathrm{p}$.

Billard, C., Alix, P., Bonnabel, L., Bonnardin, S., Bostyn, F., Caspar, J.-P., Degobertière, S., Dietsch-Sellami, M.-F., Hamon, C., Marcoux, N. et MARguerie, D., 2004 - Le site d'habitat du Néolithique ancien de Colombelles « Le Lazzaro » (Calvados) - présentation liminaire, INTERNEO, $n^{\circ} 5$, p. 29-33.

Capote, M., Castaneda, N., Consuegra, S., Criado, C. et Diaz del Rio, P., 2008 - Flint mining in early Neolithic Iberia: a preliminary report on « Casa Montero » (Madrid, Spain), in ALLARD, P. (dir.), Flint Mining in Prehistoric Europe, Interpreting the archaeological records (Actes du congrès de l'EAA, Cracovie 2006) Oxford, Archaeopress (BAR International Series, 1891), p. 123-138.

Carpentier, V., Giazzon, D., Guillier, G., Lepaumier, H., Lourdeau, C. et Marcigny, C., 2005 L'apport des fouilles extensives à la connaissance des campagnes entre Evreux et le Vieil Evreux : l'exemple de la ZAC du Long Buisson, Haute-Normandie Archéologique, n 10, p. 9-20.

Chancerel, A. et Ghesquière E., 2006 - La maison danubienne du Haut-Saint-Martin (Mondeville), in CHANCEREL, A., MARCIGNY, C. et GHESQUiÈre, E. (dir.), Le plateau de Mondeville (Calvados), du Néolithique à l'âge du Bronze, Paris, Maison des Sciences de l'Homme (DAF, 99), p. 25-53.

Coutard S. 1998 - Panorama des silex jurassiques bas-normands (mémoire de Maîtrise), Université de Caen, $83 \mathrm{p}$.

Desloges, J., 1990 - L'extraction minière du silex au Néolithique et l'exemple de Bretteville-le-Rabet (Calvados) (diplôme de l'EHESS), Toulouse, $198 \mathrm{p}$.

Desloges, J., 1999 - Une mine de silex au Néolithique, l'exemple de Bretteville-le-Rabet, L'exploitation ancienne des roches dans le Calvados, Histoire et Archéologie, Caen, Conseil Général du Calvados, p 52-77.

Desloges, J. et Ghesquière, E., 2007 - La production des lames en silex, in MARCIGNY, C., GHESQUIÈRE, E. et DESLOGES, J. (dir.), La Hache et la Meule, les premiers paysans du Néolithique en Normandie (6 000-2 000 avant notre ère), Le Havre, éd. du Muséum d'Histoire naturelle, p. 67. 
Doranlo, R., 1926 - L'archéologie antique en Normandie des origines au xe siècle. Etude bibliographique et critique sur le mouvement archéologique en Normandie pendant la période 1824-1924, Caen, Société d'Impression de Basse Normandie.

Dron, J.-L. et San Juan, G., 1992 - Ernes - Condé-sur-Ifs (Calvados) : habitat puis nécropole au Néolithique moyen. Présentation liminaire, in LE Roux, C.-T. (dir.), Paysans et bâtisseurs :

l'émergence du Néolithique atlantique et les origines du mégalithisme, Revue archéologique de l'Ouest, 5e supplément, p. 31-42.

Edeine, B., 1961 - Triple sépulture du Hallstatt final découverte en Normandie (Calvados), Bulletin de la Société préhistorique française, 58 (5-6). p. 347-359.

Edeine, B., 1963 - Contribution aux études de technologie de l'outillage néolithique. Fabrication d'outils en silex par débitage lamellaire nucléiforme dans le Danubien normand, Bulletin de la Société préhistorique française, 60 (7-8). p. 456-462.

Edeine, B., 1965a - Ce que les fouilles de la Brèche au Diable et son contexte peuvent apporter [...] à la solution des problèmes posés par G. Bailloud dans son ouvrage Le Néolithique dans le Bassin Parisien, Bulletin de la Société préhistorique française, 62, p. 328-349.

Edeine, B., 1965b - Le Néolithique de l'ouest européen en Basse-Normandie, Rubané récent, Cerny, Chasséen, Rapports interculturels et chronologie, Revista da Faculdade de Letras de Liboa, $3^{\mathrm{e}}$ série, 9 (Mélanges en l'honneur de l'abbé Breuil), p. 3-16.

Edeine, B., 1970 - Nouvelles datations par le ${ }^{14} \mathrm{C}$ concernant la Basse-Normandie, en particulier le Chasséen et le Rubané Récent, Bulletin de la Société préhistorique française, 67 (4), p. 114-120.

Edeine, B., 1972 - Nouvelles datations par le C14 concernant les sites de la Brèche au Diable (Mont Joly) et des Longrais (Calvados), Bulletin de la Société préhistorique française, 69 (CRSM, 7), p. 197-199.

Eudes-Deslongchamps, E., 1876 - Note sur les stations préhistoriques découvertes aux environs de Falaise, Mémoires de la Société des Antiquaires de Normandie, 29, p. 198-203.

Fouju, G., 1891 - Les puits préhistoriques pour l'extraction du silex de Champignolles (Oise), L'Anthropologie, 2, p. 445-455.

Galiberti, A., Di Lierna, S., Fiorentino, G. et Guarascio, M., 1998 - New data on the neolithic mine of Defensola, Vieste (Italy), in RAMOS-MILLAN \& BUSTILLO (ed.), Siliceous rocks and culture, Granada, édition de l'Université de Granada (Monografia Arte y Arqueologia 42), p. 211-222.

Ghesquière, E. et Marcigny, C., 1998 - Le débitage lithique au Cerny en Basse-Normandie, INTERNEO, $\mathrm{n}^{\circ} 2$, p. 57-68.

Ghesquière, E. et Marcigny, C., 2000 - Un village rubané en Basse-Normandie ? L'évaluation du site de la ZAC du Lazzaro à Colombelles (Calvados), Bulletin de la Société préhistorique française, 97 (3), p. 405-418.

Giraud, P. et Juhel, L., 2004 - L'habitat Villeneuve-Saint-Germain de Fontenay-le-Marmion « Le Grand Champ »(Calvados), INTERNEO, n 5, p. 35-43.

Lech, J., 1982 - Flint mining among the early farming communities of Central Europe, part. II, Przeglad Archeologiczny, 30, p. 47-80.

Marcigny, C., Desloges, J. et Ghesquière E., 2007 - De la mine à la hache, les minières de RiRonai, in DELRIEU, F. et DESLOGES, J. (dir.) - ArchéOrne, 250 ans d'archéologie dans l'Orne, Bayeux, Les Cahiers du Temps, p. 50-51. 
Marcigny, C., Ghesquière, E., Clement-Sauleau, S., Giazzon, D., Gallouin, E. et Hugot, C., 2002 - Les occupations du Néolithique moyen de Saint-Vigor-d'Ymonville (76), INTERNEO, $\mathrm{n}^{\circ} 4$, p. 37-50.

Nora, P., 1997 - Les lieux de mémoire, T.1. Paris, Gallimard.

Salmon, P., d'Ault du Mesnil, et Capitan, L., 1898 - Le Campignien, fouille d'un fond de cabane au Campigny, commune de Blangy-sur-Bresle, Revue de l'Ecole d'Anthropologie, déc. 1898.

p. $365-405$.

\section{RÉSUMÉS}

L'implantation d'un lotissement à Soumont-Saint-Quentin (Calvados) a suscité un diagnostic archéologique sur une minière de silex en partie fouillée par B. Edeine dans les années 1960. 38 puits ont ainsi pu être dégagés en surface et 9 ont fait l'objet d'une fouille archéologique. Cette minière visait à l'exploitation du silex bathonien inclus dans les argiles de recouvrement du site (dans des cuvettes de dissolution ou en nappe), ou en dessous dans le calcaire. Le débitage était réalisé in situ, comme le suggère la présence de l'intégralité de la chaîne de production piégé surtout dans le remplissage des puits. Ce débitage vise à la production par percussion indirecte de lames régulières, ainsi plus marginalement qu'à celle d'éclats courts et au façonnage de tranchets bitronqués ou bifaciaux. L'ensemble peut être daté du Néolithique ancien ou du Néolithique moyen I, ce qui fait du site des Longrais une des minières parmi les plus anciennes d'Europe du nord.

An housing development in Soumont-Saint-Quentin (Calvados) provided the opportunity for assessment on a flint mine partly excavated by B. Edeine in the ' 60.38 pits could be defined by scraping and 9 were excavated. This mine exploited bathonian flints included as well as in drift clays (in solution pockets or as seam) as in underlying limestone. Knapping was carried out in situ, as suggests evidence of the entire production process in the pit fills. Regular blades were produced by indirect percussion, but also, marginally, short flakes and bitruncated or bifacial tranchets. The whole can be dated from Early Neolithic or Middle 1 Neolithic, which makes Les Longrais one the oldest flint mines known in Northern Europe.

\section{INDEX}

Keywords : Flint Mines, Lower Normandy, Neolithic, Soumont-Saint-Quentin

Mots-clés : Basse-Normandie, Néolithique, silex, minière, Soumont-Saint-Quentin

\section{AUTEURS}

\section{JEAN DESLOGES}

DRAC/SRA de Basse-Normandie, UMR 6566-CReAAH, Université de Rennes I, Rennes II, Nantes, CNRS, MCC. (jean.desloges@culture.gouv.fr)

\section{EMMANUEL GHESQUIÈRE}

Inrap Basse-Normandie, UMR 6566-CReAAH, Université de Rennes I, Rennes II, Nantes, CNRS, MCC. (emmanuel.ghesquiere@inrap.fr) 


\section{CYRIL MARCIGNY}

Inrap Basse-Normandie, UMR 6566-CReAAH, Université de Rennes I, Rennes II, Nantes, CNRS,

MCC. (cyril.marcigny@ inrap.fr)

\section{TRADUCTEUR_DESCRIPTION}

\section{FRANÇOIS CHARRAUD (TRADUCTION)}

Doctorant, Université de Nice-Sophia Antiplois, UMR 6130-CEPAM.

(fcharraud@gmail.com) 